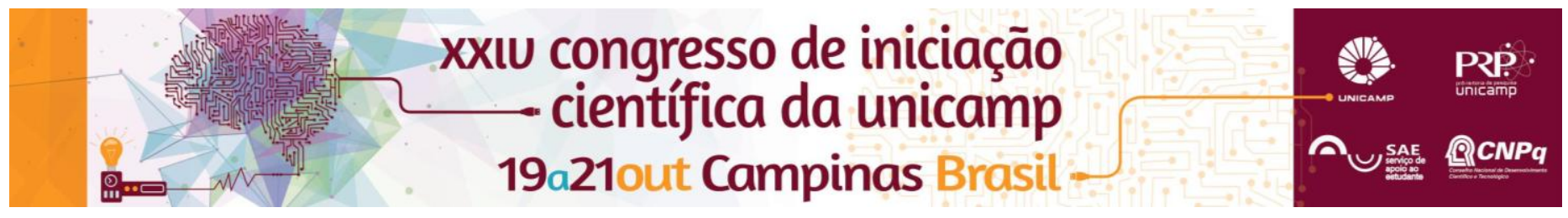

\title{
Automation of the Mapping Process in an Inverted Optical Microscope
}

\author{
Flavio A. Borges*, J.H.Clerici, Mônica A. Cotta.
}

\begin{abstract}
An inverted optical microscope allows a more natural observation of microorganism and cell cultures, in large culture plates. However, if surface mapping is carried out manually in microscopes with simpler configuration, the analysis process becomes slower. The project in question consists in the automation of the surface mapping process with the optical microscope, with the intent to optimize its duration and subsequent reconstruction of the image mosaic.
\end{abstract}

\section{Key words:}

Automation, optimization, optics microscopy.

\section{Introduction}

The inverted optical microscope has a configuration in which the light sources are placed above the stage, while the objective is placed below. ${ }^{1}$ This configuration presents the advantage of allowing the observation of microorganisms or cell cultures in a relatively large container, in a less stressful environment from a biochemical point of view. However, the translation of the stage where the sample is placed, necessary for good statistics in the experiment, takes plenty of time when made manually, in the simpler models of optical microscopes. The lack of control in the image positioning also makes more difficult the reconstruction of a large scale map of the observed surface. Thus, the optimization of the process of handling the microscope stage would be an efficient way to optimize the surface mapping process (and the objects of scientific interest within it) in such models of inverted optical microscopes. That is the main objective of this project.

\section{Results and Discussion}

The automation project can be divided in three steps: the mechanical project, the electrical project and programming and control.

The mechanical project was necessary to make an addition of two step motors in the best way possible, keeping the movement quality in the two axes to be controlled. In our case, the most suitable geometry for the motorized stage was chosen to be used with an inverted microscope Nikon Eclipse TE2000-U, at the Laboratory of Nano and Biosystems, IFGW, UNICAMP.

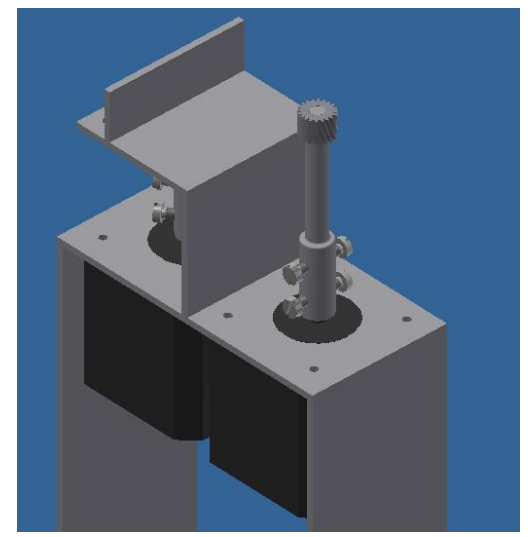

Image 1. Mechanical project of the movement mecanism made using the sotware Autodesk Inventorß.
The electrical project was necessary to install the driver for the manipulation of the number of steps of each motor. With that we will be able to have movement control of the axys in the same scale (in micrometers) used in the sample analysis with the microscope.

For the programming and control of the process, we used the software Labview®. This step was necessary so that the movement with the step motors is precise, considering the movement of an analisys process in the microscope. Moreover, secure measures were made and implemented to keep both microscope and components as safe as possible.

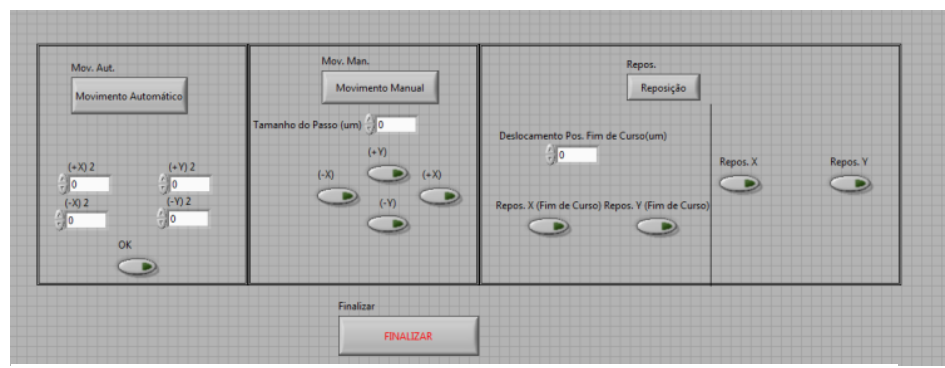

Image 2. Control panel on Labview ${ }^{\circledR}$ with options of manual and automatic control, and repositioning to the inicial configuration.

\section{Conclusions}

We show here the automation of the analysis process of surface mapping (for microorganism adhesion evaluation) in an inverted optical microscope. The automation allowed a significant improvement in the process regarding duration and ease of use.

\section{Acknowledgement}

We acknowledge Rafael Hensel for the initial studies in this project, on which we based the development carried out in this work. We also also acknowledge financial support from $\mathrm{CNPq}$ for the undergraduate researcher scholarship (grant process 479486/2012-3).

\footnotetext{
More detailed information can be found, for example, a https://www.nikoninstruments.com/pr_BR/Produtos/Microscopios-Invertidos (access in 17/July/2016).
} 